This item was submitted to Loughborough's Research Repository by the author.

Items in Figshare are protected by copyright, with all rights reserved, unless otherwise indicated.

\title{
Warm discharges in cold fresh water: 2. Numerical simulation of laminar line plumes
}

PLEASE CITE THE PUBLISHED VERSION

http://dx.doi.org/10.1007/s10652-016-9468-x

\section{PUBLISHER}

(c) Springer

VERSION

AM (Accepted Manuscript)

\section{PUBLISHER STATEMENT}

This work is made available according to the conditions of the Creative Commons Attribution-NonCommercialNoDerivatives 4.0 International (CC BY-NC-ND 4.0) licence. Full details of this licence are available at: https://creativecommons.org/licenses/by-nc-nd/4.0/

\section{LICENCE}

CC BY-NC-ND 4.0

\section{REPOSITORY RECORD}

George, Alabodite M., and Anthony Kay. 2019. "Warm Discharges in Cold Fresh Water: 2. Numerical Simulation of Laminar Line Plumes". figshare. https://hdl.handle.net/2134/21755. 


\title{
Warm discharges in cold fresh water: 2. Numerical simulation of laminar line plumes
}

\author{
Alabodite M. George · Anthony Kay
}

Received: date / Accepted: date

\begin{abstract}
The behaviour of a discharge of warm water upwards into a homogeneous body of cold fresh water was investigated by means of a numerical model. The discharge has a parabolic velocity profile, with Reynolds number $\operatorname{Re}=50$, Prandtl number $\operatorname{Pr}=7$ and Froude number varied over the range $0.2 \leq F r \leq 2.5$. Water density is taken to be a quadratic function of temperature, so that an initially positively buoyant discharge will experience buoyancy reversal as it mixes with an ambient below the temperature of maximum density. The resulting plume has some similarities to a fountain resulting from injection of negatively buoyant fluid upward into a less dense ambient. The plume is initially symmetric, but then its head detaches as it approaches its maximum height. The detached head is denser than the fluid in the plume below it, and the interaction between the sinking head and the rising plume causes a sideways deflection; as this cycle is repeated, the plume displays side-toside flapping motion and vertical bobbing. As Froude number is increased (i.e. buoyancy reduced) the growth of the plume becomes slower, but the plume eventually reaches a greater height. We obtain empirical power-law scalings for maximum height and time taken to reach that height as functions of Froude number; these scalings are simlar to those for fountains with a linear dependence of density on temperature in the very weak regime.
\end{abstract}

Keywords Cold water $\cdot$ Line plume $\cdot$ Buoyancy reversal $\cdot$ Numerical simulation

\section{Introduction}

Buoyant plumes in cold fresh water are of interest because of the possibility of buoyancy reversal due to the nonlinear relation between temperature and density in water. Consider a discharge of warm water at the bed of a lake with an ambient temperature below the temperature of maximum density, which is approximately $4{ }^{\circ} \mathrm{C}$ in fresh water. Mixing of the warm water with the ambient can then produce water that is denser than both the warm discharge and the ambient water, the process termed "cabbeling" by Foster [1]. Thus, a plume of warm

Anthony Kay

Department of Mathematical Sciences, Loughborough University, Loughborough, Leicestershire, LE11 3TU, United Kingdom

Tel.: +44-1509-222878

E-mail: A.Kay@lboro.ac.uk 


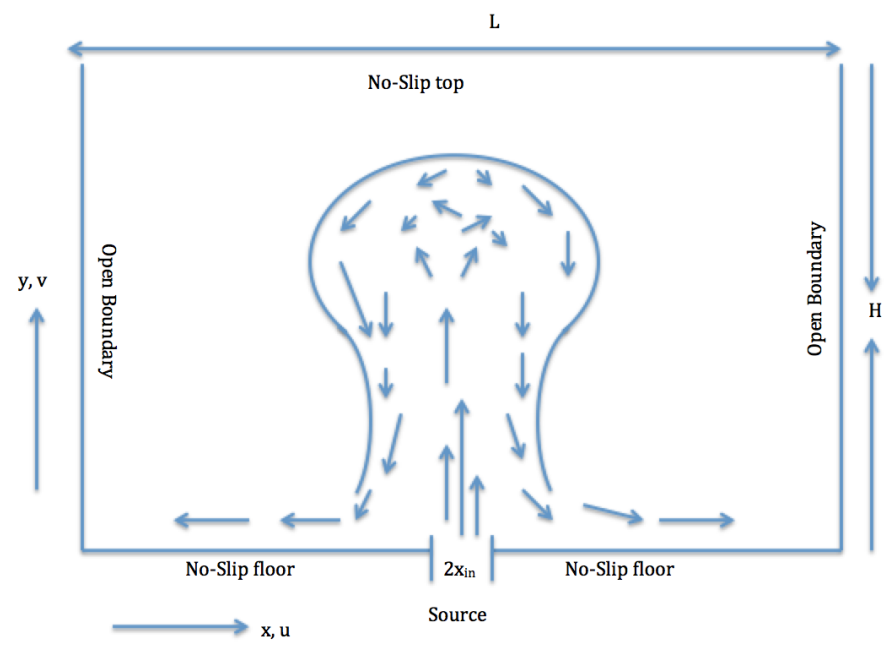

Fig. 1 Schematic of plume with buoyancy reversal, also showing domain for computations

water which initially rises due to positive buoyancy may experience buoyancy reversal and become a fountain as dense mixed water falls back towards the source: see Figure 1. Alternatively, the initial buoyancy and momentum of the plume may be sufficient to carry it to the surface of the lake, where it will spread as a gravity current if still positively buoyant; but further entrainment of cold water into the gravity current will then lead to buoyancy reversal as a result of cabbeling, and the gravity current will be arrested and its dense water will then sink to the lake bed: see Figure 2.

Power station cooling water discharges are one example of a situation where flows of this type may occur. The cooling water is typically discharged at a temperature approximately $10^{\circ} \mathrm{C}$ above that of the receiving water [2,3]. Thus the discharge will certainly be less dense than the ambient, so will initially form a rising plume; but if the ambient is below the temperature of maximum density, buoyancy reversal due to cabbeling will occur. Mixed water close to the temperature of maximum density will then descend to the lake bed: for example, Hoglund and Spigarelli [4] observed water at a temperature of $5.7^{\circ} \mathrm{C}$ at the bed of Lake Michigan in the vicinity of a power station outfall while the natural ambient temperature was $0.5^{\circ} \mathrm{C}$, and surmised that the relatively warm but dense water had been formed by this mechanism.

While power station discharges will obviously be fully turbulent, laboratory experiments such as those of Bukreev and Gusev [5] yield plumes at much lower Reynolds numbers, and the flow may be laminar or in transition to turbulence. The focus of the present paper is laminar plumes which do not reach the surface of the ambient water body, but form fountains due to buoyancy reversal. Previous authors have done much analysis of both turbulent and laminar fountains in the more usual situation of linear dependence of density on temperature, with a negatively buoyant fluid being injected upwards into a less dense ambient. Lin and Armfield [6,7] used computational models for axisymmetric and two-dimensional laminar 


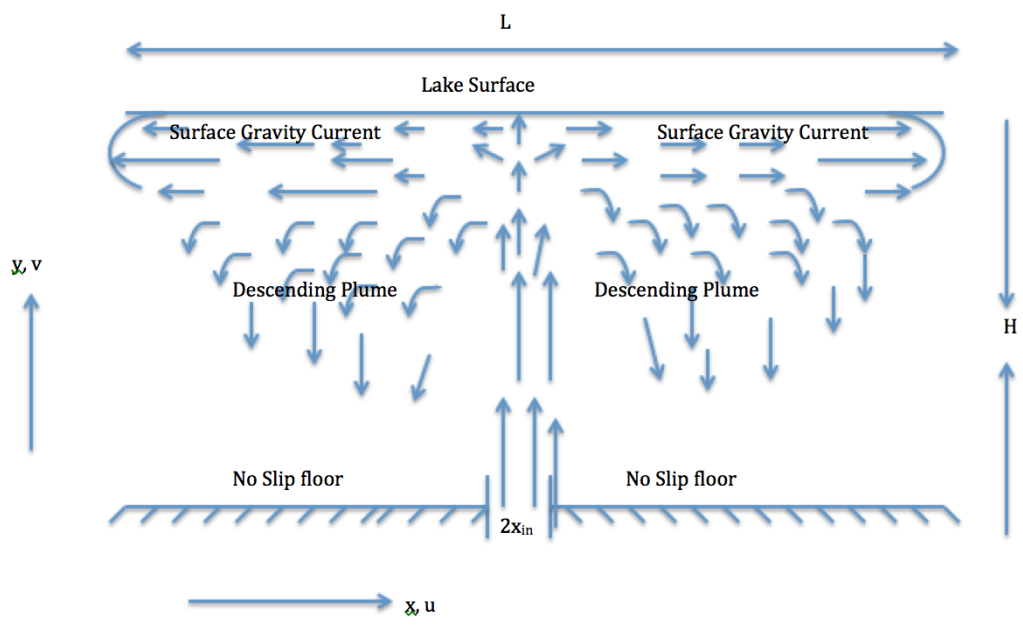

Fig. 2 Schematic of plume reaching lake surface before experiencing buoyancy reversal

fountains to determine linear relations of the form $z_{m} / x_{0}=a_{0}+a_{1} F r$ between the final, steady fountain height $z_{m}$ and the source Froude number $F r=V_{0} /\left(x_{o} g_{0}^{\prime}\right)^{1 / 2}$ in the range $0.2 \leq F r \leq 1.0$. Here $x_{0}$ is the half-width of a line source or the radius of an axisymmetric source, $V_{0}$ and $g_{0}^{\prime}$ are respectively the mean velocity and reduced gravity at the source, and the numerical coefficients $a_{0}$ and $a_{1}$ depend on the source geometry (axisymmetric or line source) and velocity profile. They also found similar linear relations for the fountain width, and quadratic relations of the form $t_{m} /\left(x_{0} / V_{0}\right)=b_{0}+b_{2} F r^{2}$ for the time $t_{m}$ taken to establish the steady fountain height. These computations were for fixed Reynolds number $R e=200$ and Prandtl number $\operatorname{Pr}=7$; subsequent computations in which these parameters were varied [8] indicated that $z_{m} / x_{0}=a_{0}+a_{1} F r \operatorname{Re}^{-1 / 2}$ (and similarly for fountain width) as long as $R e \leq 200$, but that fountain height and width became independent of Reynolds number at larger values of $R e$. When the range of Froude numbers was extended up to $F r=$ 10 and the symmetry constraint in the computational domain was removed, Srinarayana et al. [9] found that the fountain remained steady and symmetric only up to $F r \approx 2.25$. At higher Froude numbers, lateral oscillations ("flapping") were found; these were periodic for $F r \leq 4.0$ but chaotic for $F r>4.0$. The scaling for the fountain height was $z_{m} / x_{0} \sim$ $F r^{1.15}$ in the periodic oscillation regime, and $z_{m} / x_{0} \sim F r^{4 / 3}$ with chaotic flapping. These results were obtained with a uniform velocity profile at the fountain source; subsequently, Srinarayana, Armfield and Lin [10] assumed a parabolic source velocity profile and obtained different values of the transition Froude numbers, and a $\mathrm{Fr}^{2}$ scaling for fountain height in the periodic flapping regime. Laboratory experiments by Williamson et al. [11] and Srinarayana et al. [12] revealed a broader range of fountain behaviours, and determined how transitions between the behaviours depended on both Froude and Reynolds numbers. Most recently, a very thorough experimental investigation of the dependence of fountain height on both Froude and Reynolds numbers has been reported by Burridge, Mistry and Hunt [13]. 
While the above studies have all considered flows in which the buoyancy flux opposes the momentum flux at the source, there has been less attention given to fountains arising from buoyancy reversal. In experiments with fully turbulent flows, Turner [14] found smallamplitude, chaotic oscillations of the fountain top in the former case, but large-amplitude, periodic oscillations in the latter. Buoyancy reversal requires a nonlinear relation between density and the mixing ratio of discharged and ambient fluids: assuming density to be a quadratic function of mixing ratio, with the mixed fluid less dense than the mean of the constituents' densities, Caulfield and Woods [15] used the entrainment assumption of Morton, Taylor and Turner [16] in a model of a dense plume injected upwards into a less dense ambient. This is equivalent to warm water being injected downwards into an ambient colder than the temperature of maximum density, in which case the plume may form an inverted fountain or may continue downwards without being arrested, depending on the initial conditions. Kay [17] analysed this case as well as the upwards injection of warm water into a cold ambient, again using the classical entrainment assumption. The problem with this approach is that it can model a plume as far as the level where it is arrested, but cannot account for the reversed flow in a fountain; furthermore, the flow was assumed steady by both Caulfield and Woods [15] and Kay [17]. Experiments by Bukreev and Gusev [5] involved the injection of warm water upwards into an ambient below the temperature of maximum density at low Reynolds number, so that a laminar fountain was produced; as in the turbulent case studied by Turner [14], they noted periodic oscillations of the fountain height, and they also found that at certain times the descending dense water would be displaced to one side of the rising plume. However, they only presented a few examples of experimental results, without any systematic investigation of how the flow depends on source conditions. M.M.Scase (pers. comm.) has also reported a similar experiment in which a laminar fountain was produced in this way, although the temporal variations in fountain height were quite small.

The purpose of the present article is to report the results of numerical simulations of laminar fountains resulting from buoyancy reversal. The ambient is assumed to be a quiescent and homogeneous body of water, colder than the temperature of maximum density. Warm water, initially less dense than the ambient, is injected at constant speed from a line orifice in the base of the container, so the flow is assumed two-dimensional. We keep the Reynolds and Prandtl numbers the same in all simulations, $\operatorname{Re}=50$ and $\operatorname{Pr}=7$, and vary the Froude number which represents the balance between inertia and buoyancy forces. Details of the model are given in Section 2, the results of our simulations are presented in Section 3 , and we draw some conclusions in Section 4.

\section{Model Formulation and Governing Equations}

The nonlinear relationship between density $\rho$ and temperature $T$ is fundamental to our study. We assume a quadratic relation,

$$
\rho=\rho_{m}-\beta\left(T-T_{m}\right)^{2},
$$

which provides a good fit to the experimentally determined density of fresh water at temperatures below $10^{\circ} \mathrm{C}$ if we take $T_{m}=3.98^{\circ} \mathrm{C}, \rho_{m}=1.000 \times 10^{3} \mathrm{~kg} \cdot \mathrm{m}^{-3}$ and $\beta=8.0 \times$ $10^{-3} \mathrm{~kg} \cdot \mathrm{m}^{-3}\left({ }^{\circ} \mathrm{C}\right)^{-2}[18,19]$. Other fluid properties (e.g. viscosity, thermal diffusivity) are assumed constant.

Our computational study uses a rectangular domain of height $H=30 x_{\text {in }}$ and width $L=$ $60 x_{\text {in }}$, with open sides and rigid, no-slip top and base, except for an orifice of width $2 x_{\text {in }}$ in the centre of the base, through which warm fluid is injected at mean velocity $v_{\text {in }}$ : see Figure 
1. The velocity profile at this plume source is assumed parabolic as for laminar Poiseuille flow (cf. [10]),

$$
v(x, 0)=\frac{3}{2} v_{\text {in }}\left[1-\left(\frac{x}{x_{\text {in }}}\right)^{2}\right] .
$$

The temperature $T_{\text {in }}$ of this injected fluid is constant, and the initial ambient temperature $T_{\infty}$ is uniform.

To define dimensionless variables, the difference between the ambient and the temperature of maximum density provides a natural temperature scale, while the source conditions provide the only length and velocity scales; the domain dimensions are not suitable length scales, as they are taken to be large enough for conditions at the sides and top of the domain to have no effect on the plume. Hence, we non-dimensionalise the coordinates $x, y$, velocity components $u, v$, time $t$, pressure $p$ and temperature $T$ by

$$
U=\frac{u}{v_{\text {in }}} \quad V=\frac{v}{v_{\text {in }}} \quad X=\frac{x}{x_{\text {in }}} \quad Y=\frac{y}{x_{\text {in }}} \quad \tau=\frac{t}{x_{\text {in }} / v_{\text {in }}} \quad P=\frac{p}{\rho v_{\text {in }}^{2}} \quad \phi=\frac{T-T_{\infty}}{T_{m}-T_{\infty}},
$$

where $x$ and $u$ are horizontal, $y$ and $v$ are vertical. We also define dimensionless parameters, the Reynolds, Prandtl and Froude numbers, by

$$
R e=\frac{v_{i n} x_{i n}}{v}, \quad \operatorname{Pr}=\frac{v}{\alpha}, \quad F r^{2}=\frac{\rho_{m} v_{i n}^{2}}{g \beta\left(T_{m}-T_{\infty}\right)^{2} x_{i n}}
$$

where $v$ and $\alpha$ are the respective diffusivities of momentum and heat, $v=\mu / \rho$ and $\alpha=$ $k / \rho c_{p}$ where $\mu$ is viscosity, $k$ is thermal conductivity and $c_{p}$ is specific heat.

In terms of these dimensionless variables and parameters, the continuity equation, the horizontal and vertical momentum equations and the thermal energy equation are

$$
\begin{gathered}
\frac{\partial U}{\partial X}+\frac{\partial V}{\partial Y}=0 \\
\frac{\partial U}{\partial \tau}+U \frac{\partial U}{\partial X}+V \frac{\partial U}{\partial Y}=-\frac{\partial P}{\partial X}+\frac{1}{R e}\left(\frac{\partial^{2} U}{\partial X^{2}}+\frac{\partial^{2} U}{\partial Y^{2}}\right) \\
\frac{\partial V}{\partial \tau}+U \frac{\partial V}{\partial X}+V \frac{\partial V}{\partial Y}=-\frac{\partial P}{\partial Y}+\frac{1}{R e}\left(\frac{\partial^{2} V}{\partial X^{2}}+\frac{\partial^{2} V}{\partial Y^{2}}\right)+\frac{1}{F r^{2}}\left(\phi^{2}-2 \phi\right) \\
\frac{\partial \phi}{\partial \tau}+U \frac{\partial \phi}{\partial X}+V \frac{\partial \phi}{\partial Y}=\frac{1}{\operatorname{RePr}}\left(\frac{\partial^{2} \phi}{\partial X^{2}}+\frac{\partial^{2} \phi}{\partial Y^{2}}\right) .
\end{gathered}
$$

The only unusual term here is the buoyancy term (the last term in the vertical momentum equation), the form of which is determined by the quadratic equation of state (1).

Our initial conditions are an undisturbed, homogeneous medium,

$$
U=0, \quad V=0, \quad \phi=0 \text { for } \tau<0 .
$$

For $\tau \geq 0$ we have boundary conditions as follows. On the side walls:

$$
\frac{\partial U}{\partial X}=0, \quad \frac{\partial V}{\partial X}=0, \quad \frac{\partial \phi}{\partial X}=0 \quad \text { at } \quad X= \pm \frac{L}{2 x_{\text {in }}} .
$$

At the plume source in the base of the domain:

$$
U=0, \quad V(X, 0)=1.5\left(1-X^{2}\right), \quad \phi=\phi_{\text {in }} \quad \text { for } \quad|X| \leq 1 \quad \text { at } \quad Y=0 .
$$


Elsewhere on the base:

$$
U=0, \quad V=0, \quad \frac{\partial \phi}{\partial Y}=0 \quad \text { for } \quad|X|>1 \quad \text { at } \quad Y=0 .
$$

At the top of the domain:

$$
U=0, \quad V=0, \quad \frac{\partial \phi}{\partial Y}=0 \quad \text { at } \quad Y=\frac{H}{x_{\text {in }}} .
$$

The Reynolds number $R e=50$ and Prandtl number $\operatorname{Pr}=7$ will be fixed throughout this study, as will the dimensionless inlet temperature $\phi_{\text {in }}=2.5$ which is equivalent to a discharge at $10^{\circ} \mathrm{C}$ into an ambient at $0^{\circ} \mathrm{C}$. However, the Froude number will be varied. If we suppose the length scale to be fixed, the Reynolds number fixes the source velocity and the Froude number is then inversely related to the buoyancy; thus the magnitude of buoyancy will be varied, while the value of $\phi_{\text {in }}$ fixes the relative buoyancies of source and ambient with respect to water at maximum density.

Numerical solution of the above equations is by means of COMSOL Multiphysics software. This commercial package uses a finite element solver with discretization by the Galerkin method and stabilisation to prevent spurious oscillations. We have used the "Extra fine" setting for the mesh. Time stepping is by COMSOL's Backward Differentiation Formulas. Further information about the numerical methods is available from the COMSOL Multiphysics website [20].

Results will be illustrated mainly by contour plots of dimensionless temperature on a colour scale from dark red for the ambient temperature $\phi=0.0$, through yellow to white for the source temperature $\phi=2.5$. Note that $\phi=1.0$ corresponds to the temperature of maximum density while $\phi=2.0$ is the temperature at which warm water has the same density as the ambient cold water. The contour plots show the entire domain of computation, $-30 \leq X \leq 30,0 \leq Y \leq 30$.

\section{Results}

Figures $3-9$ follow the progress of four plumes, with differing Froude numbers $\mathrm{Fr}=$ $0.2,0.5,1$ and 2.5 (shown in panels (a), (b), (c), (d) respectively of the figures), but with other flow parameters fixed as specified above. At dimensionless time $\tau=1$ (Figure 3), the plume with the lowest Froude number (greatest buoyancy) has risen furthest but has already nearly attained its maximum height (compare panel (a) in subsequent figures). Plumes with higher source Froude numbers rise more slowly, but attain greater maximum heights. All plumes eventually stop rising, having begun to lose momentum when the positive buoyancy in the interior of the plume is overcome by the negative buoyancy of the mixed water at its periphery. Figure 3 (a) shows that at $\tau=1$ a significant quantity of dense fluid has already been shed from the outside of the plume with $F r=0.2$ and is accumulating at the bed. Figure 4 shows the mixing being less vigorous for plumes with higher Froude numbers, accounting for their ultimate rise to greater heights.

All the plumes are still symmetric at $\tau=1.6$ (Figure 4). The more slowly rising plumes retain their symmetry beyond $\tau=5$, at which time they have developed a "mushroom" structure with a vortex on either side of the plume centre-line. However, all plumes eventually lose their symmetry through a process of "head detachment" (Figures 5 (b), 6 (c), 7 (d)). The head of the plume becomes denser than the neck beneath it, and so rises more slowly. The subsequent interaction between the head and the faster rising fluid in the neck causes 


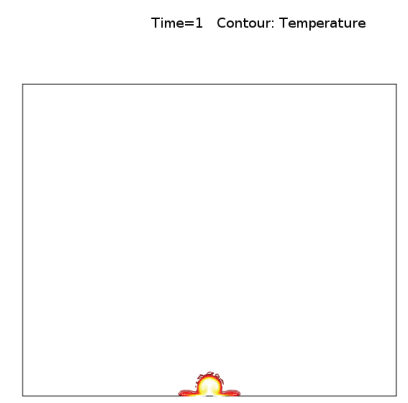

(a) $F r=0.2$

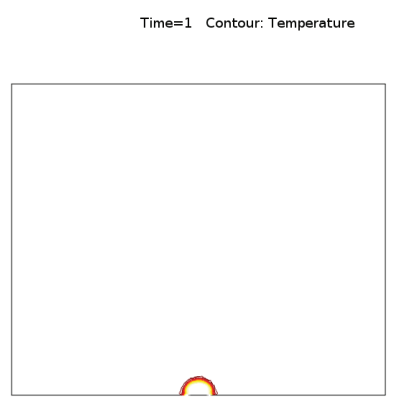

(c) $F r=1.0$
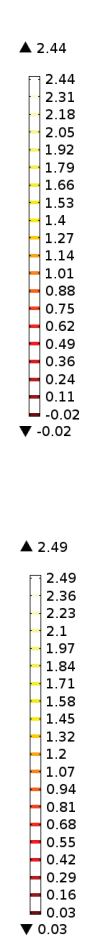

Time=1 Contour: Temperature

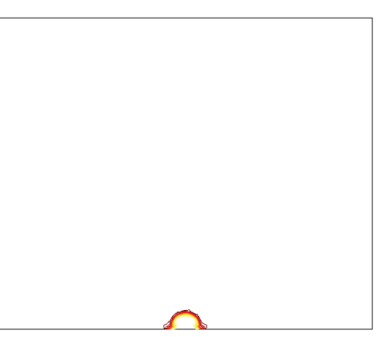

(b) $F r=0.5$
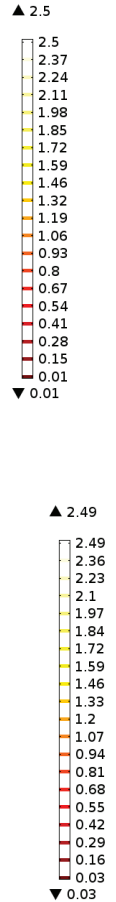

Fig. 3 Temperature field at time $\tau=1$ for plumes with $\operatorname{Re}=50, \operatorname{Pr}=7, \phi_{\text {in }}=2.5$ and (a) $F r=0.2$, (b) $F r=0.5$, (c) $F r=1$ and (d) $F r=2.5$

the head to be deflected to one side or the other, and then to detach and sink to the bed. As the production of positively buoyant fluid from the source and the production of dense fluid by mixing are continuous processes, a continuous "flapping" and "bobbing" of the plume develops: the sideways deflection of detaching heads results in side-to-side flapping, and the exposure of the rising core of the plume after each head is detached leads to vertical bobbing. This behaviour has some similarities to that observed by Srinarayana et al [9] for a laminar plane fountain with $F r=8$ and a linear dependence of density on temperature; they also observed flapping from side to side as the top section of the fountain was repeatedly detached. A vertical bobbing behaviour has been previously observed by Turner [14] in a turbulent plume with reversing buoyancy, while a combination of flapping and bobbing has been seen by Vinoth and Panigrahi [21] in non-Boussinesq laminar fountains at higher Froude numbers.

Dense fluid reaching the floor spreads sideways as a gravity current with the elevated head typical of such flows [22], as seen in Figures 5(b), 6(c) and 7(d). This horizontal motion is rather slow due to the small density difference between the current and the ambient. The spread of the current is steady except when it is perturbed by the arrival of new dense fluid from a detached head, which may cause oscillations downstream.

Further insight can be gained by examining profiles of temperature and velocity components up the centre-line of the plume $(X=0)$. Figures $10-12$ show these profiles for the four Froude number cases and at the five times shown in Figures $5-9$. The temperature profiles in Figure 10 confirm that plumes with higher Froude number penetrate to greater 


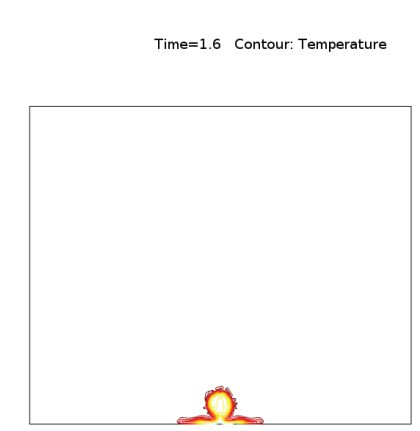

(a) $F r=0.2$

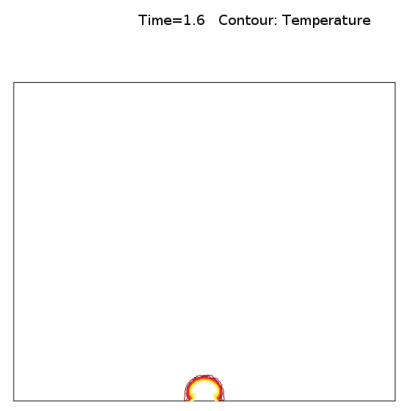

(c) $F r=1.0$
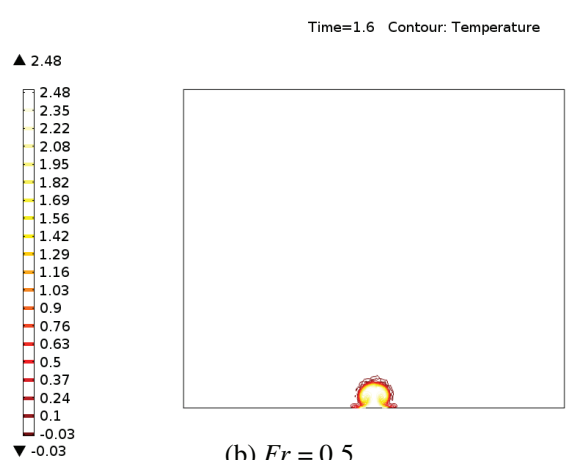

(b) $F r=0.5$

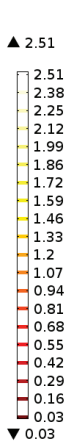

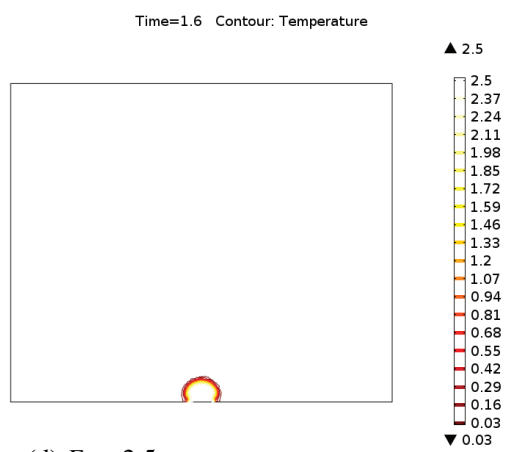

(d) $F r=2.5$

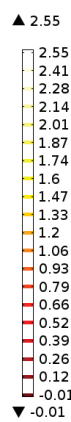

Fig. 4 Temperature field at time $\tau=1.6$ for plumes with $\operatorname{Re}=50, \operatorname{Pr}=7, \phi_{\text {in }}=2.5$ and (a) $F r=0.2$, (b) $F r=0.5$, (c) $F r=1$ and (d) $F r=2.5$

heights, and reach maximum height at later times, as seen in Figures $3-9$. The height of all plumes decreases following maximum penetration. In more detail, there is a region of almost uniform temperature immediately above the source (except for the smallest Froude number). Above this, the decrease in temperature with height is not monotonic: there are fluctuations in the temperature, which then reaches a local maximum before decreasing sharply to the ambient temperature at the top of the plume. The intense mixing within the plumes is indicated by the fluctuations in temperature; nevertheless, the most buoyant (warmest) fluid always rises to the top.

Profiles of horizontal velocity component up the plume centre-line are shown in Figure 11. Even at the earliest time shown $(\tau=5)$, there is strong side-to-side motion close to the source in the cases of $F r=0.2$ and $F r=0.5$ (Figures 11 (a), (b)). At higher Froude numbers (Figures 11 (c), (d)), the sideways motion takes longer to develop and is less intense but extends to greater heights. The profiles of vertical velocity component (Figure 12) also show intense fluctuations near the source at the lowest Froude number, with substantial downward velocities recorded at some times. At higher Froude numbers the decrease in vertical velocity with height is smoother, but still involves some degree of fluctuation; downward velocities are found in the upper part of the plume after it has reached its maximum height, as the head detaches and starts to sink.

The maximum height $Z_{n}$ attained by the plume and the time $\tau_{n}$ taken to attain that height are tabulated for a range of Froude numbers up to 2.5 in Table 1, and plotted in Figures 13 


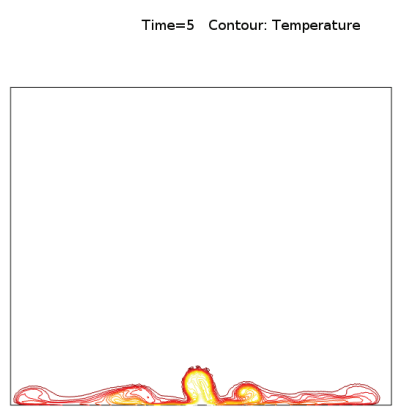

(a) $F r=0.2$
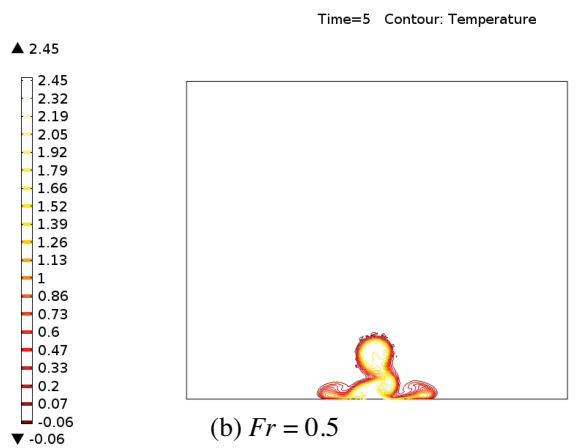

(b) $F r=0.5$
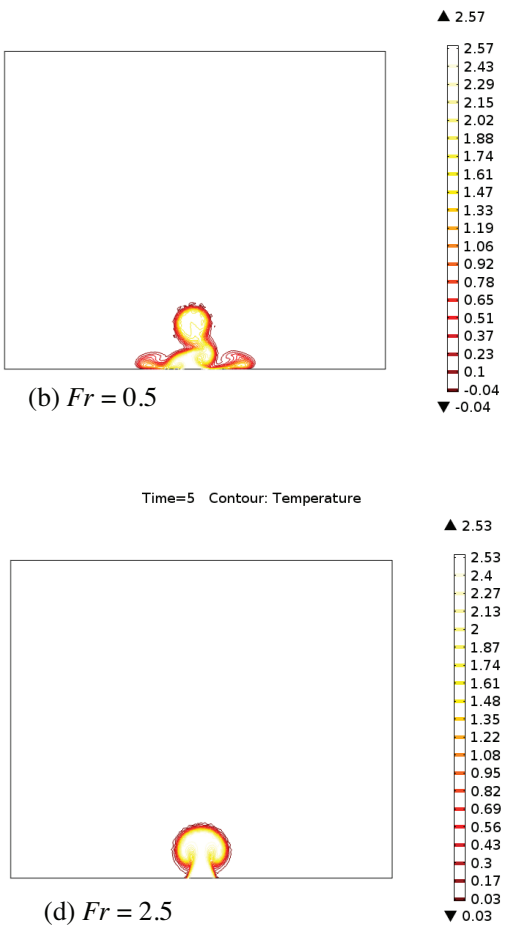

(c) $\mathrm{Fr}=1.0$

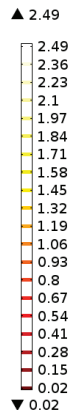

Fig. 5 Temperature field at time $\tau=5$ for plumes with $\operatorname{Re}=50, \operatorname{Pr}=7, \phi_{\text {in }}=2.5$ and (a) $F r=0.2$, (b) $F r=0.5$, (c) $F r=1$ and (d) $F r=2.5$

and 14. Srinarayana et al. [9] identified three regimes of Froude number dependence for the height of their fountains, reflecting qualitative differences in behaviour (e.g. between steady and unsteady flow) in different ranges of $F r$. In contrast, we have not found such differences in behaviour, and our data only permit us to identify a single regime of $F r$-dependence with any confidence. This is shown by the straight lines in Figures 13 and 14, which represent best fit power laws obtained by linear regression of $\log Z_{n}$ and $\log \tau_{n}$ on $\log F r$ :

$$
\begin{array}{ll}
Z_{n}=9.286 F r^{0.755} & {\left[R^{2}=0.9961\right]} \\
\tau_{n}=11.20 F r^{1.174} & {\left[R^{2}=0.9975\right],}
\end{array}
$$

where $R^{2}$ is the regression coefficient in each case. There is some suggestion in Figures 13 and 14 that larger values of the exponent might be appropriate for both relations when $F r \gtrsim 1.5$, but not sufficient evidence to justify proposing a separate regime.

\section{Conclusion}

We have investigated laminar plumes with a quadratic dependence of density on temperature by means of a computational model. With dimensionless source temperature $\phi_{\text {in }}=2.5 \mathrm{a}$ 


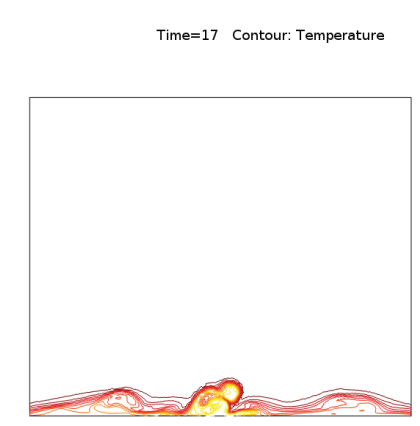

(a) $F r=0.2$

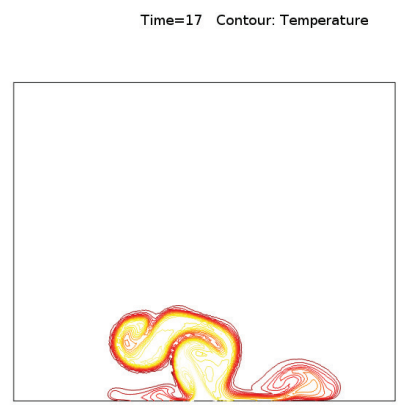

(c) $\mathrm{Fr}=1.0$
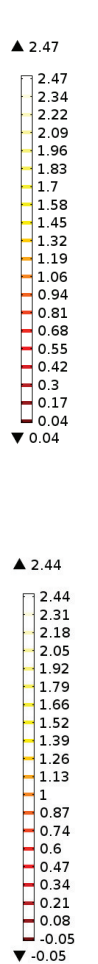

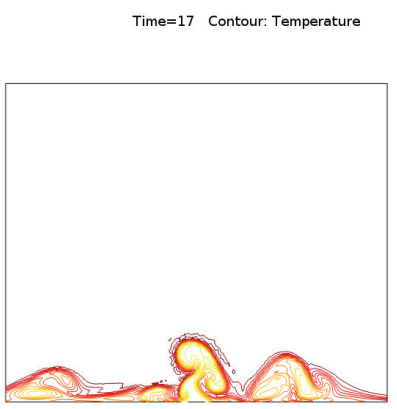

(b) $F r=0.5$

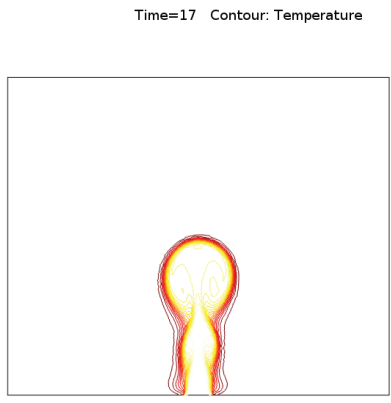

(d) $F r=2.5$
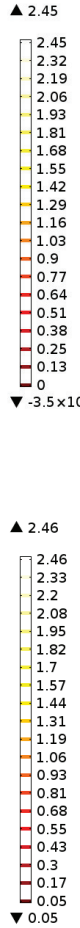

Fig. 6 Temperature field at time $\tau=17$ for plumes with $R e=50, P r=7, \phi_{\text {in }}=2.5$ and (a) $F r=0.2$, (b) $F r=0.5$, (c) $F r=1$ and (d) $F r=2.5$

Table 1 Maximum plume height $Z_{n}$ and time taken to reach that height at Froude numbers $0.1 \leq F r \leq 2.5$

\begin{tabular}{||ccc|ccc|ccc||}
\hline \hline Fr & $\tau_{n}$ & $Z_{n}$ & Fr & $\tau_{n}$ & $Z_{n}$ & Fr & $\tau_{n}$ & $Z_{n}$ \\
\hline \hline 0.1 & 0.8 & 1.873 & 1.0 & 10.5 & 9.279 & 1.9 & 24.5 & 15.17 \\
\hline 0.2 & 1.9 & 2.660 & 1.1 & 12.4 & 10.00 & 2.0 & 26 & 15.83 \\
\hline 0.3 & 2.5 & 3.550 & 1.2 & 12.8 & 10.21 & 2.1 & 27.2 & 16.38 \\
\hline 0.4 & 3.8 & 4.427 & 1.3 & 14.4 & 10.97 & 2.2 & 29.5 & 17.14 \\
\hline 0.5 & 4.5 & 5.231 & 1.4 & 16 & 11.71 & 2.3 & 30.5 & 17.70 \\
\hline 0.6 & 6.2 & 6.262 & 1.5 & 18.3 & 12.29 & 2.4 & 32.5 & 18.25 \\
\hline 0.7 & 7.2 & 6.976 & 1.6 & 19.8 & 13.44 & 2.5 & 33.5 & 19.34 \\
\hline 0.8 & 8.6 & 7.933 & 1.7 & 21.3 & 14.29 & & & \\
\hline 0.9 & 9.8 & 8.506 & 1.8 & 23 & 14.98 & & & \\
\hline
\end{tabular}

fairly small degree of mixing is required to achieve buoyancy reversal (at $\phi=2$ ). The mixing is most vigorous in plumes with the smallest Froude number (greatest buoyancy), which rise fastest initially but are brought to rest most rapidly as the mixing generates negatively buoyant fluid. Plumes with less buoyancy rise more slowly, are subject to slower mixing, and so reach greater heights before sufficient negatively buoyant fluid is produced to halt their rise. 


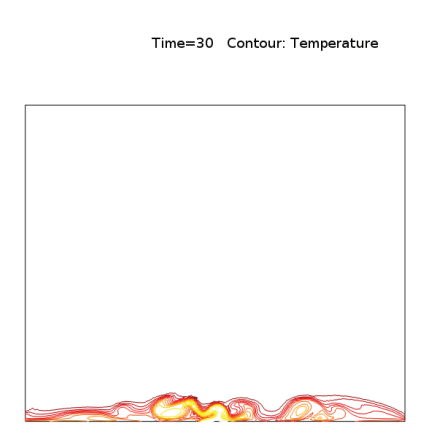

(a) $F r=0.2$

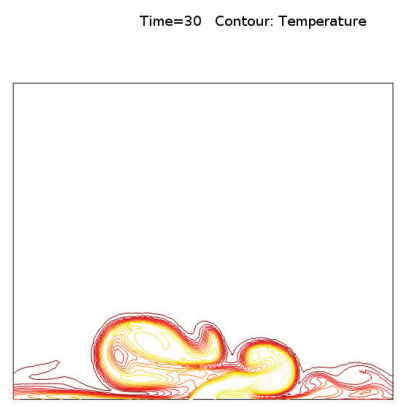

(c) $F r=1.0$
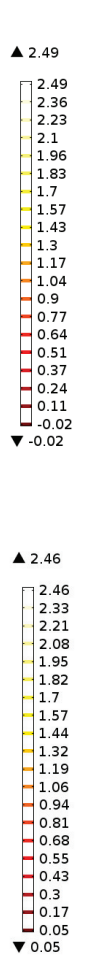

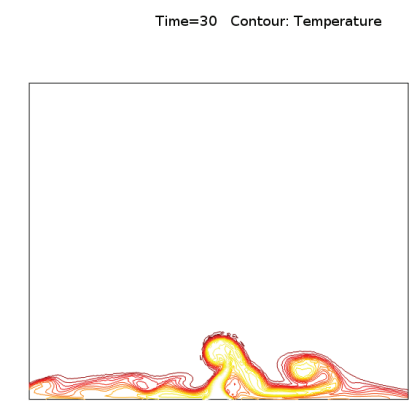

(b) $F r=0.5$

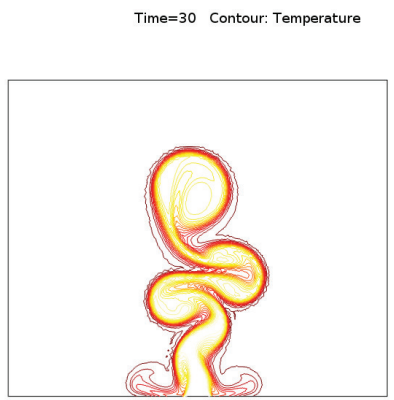

(d) $F r=2.5$
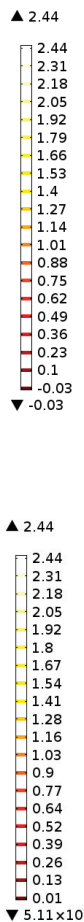

Fig. 7 Temperature field at time $\tau=30$ for plumes with $\operatorname{Re}=50, \operatorname{Pr}=7, \phi_{\text {in }}=2.5$ and (a) $F r=0.2$, (b) $F r=0.5$, (c) $F r=1$ and (d) $F r=2.5$

Our empirical Froude number dependences in equations (14) and (15) are fairly similar to the scalings for fountain height and time to steady state proposed by Lin and Armfield [23] for very weak fountains, $Z_{n} \propto F r^{2 / 3}$ and $\tau_{n} \propto F r^{4 / 3}$. A fountain is defined to be very weak if inertia effects are negligible, so that there is a balance between buoyancy and viscous forces; thus a fountain is very weak if $F r \ll 1$, whereas we have found (14) and (15) to apply for Froude numbers up to about 2. A possible explanation for this is as follows. The mechanism for halting the rise of the plume differs between our case and that of laminar fountains: whereas fountains have negative buoyancy regardless of any mixing, in our plumes negative buoyancy only appears as the consequence of mixing. With a laminar plume, that mixing is by thermal conduction, and with fixed Prandtl number the thermal conductivity is a fixed multiple of the viscosity. So the plume height may be determined by a balance between buoyancy and conduction even when inertia is not negligible, and this balance will yield the same Froude number scaling as the buoyancy-viscosity balance in very weak fountains.

As well as recording plume heights, we have examined the behaviour of the plumes in detail. Although plumes are initially symmetric, they subsequently display sideways flapping and vertical bobbing, which appear to be a consequence of the head detaching as it approaches its maximum height. The head at this stage is denser than the plume fluid below, and displaces the plume sideways as it sinks, after which the exposed core of the plume can rise again. After reaching the floor, the dense mixed fluid spreads sideways as a gravity current. 


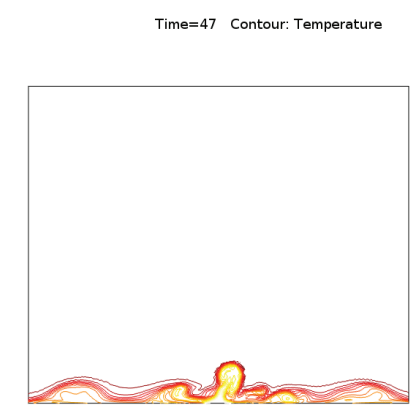

(a) $F r=0.2$

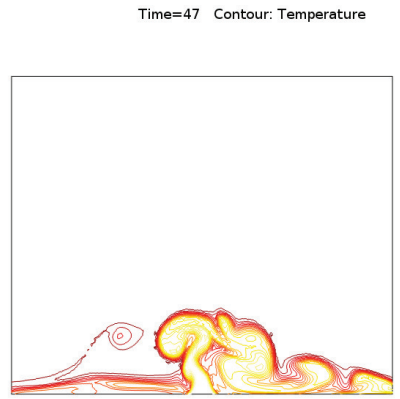

(c) $\mathrm{Fr}=1.0$
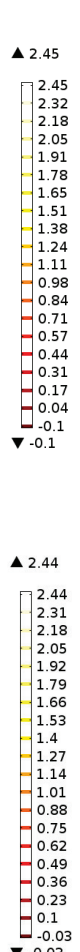

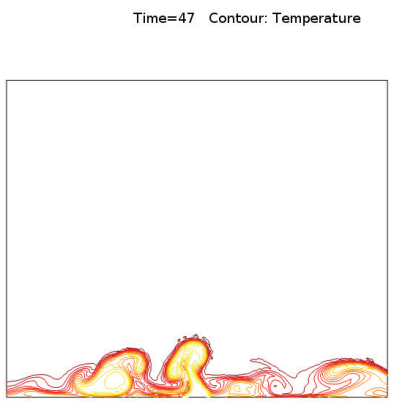

(b) $F r=0.5$

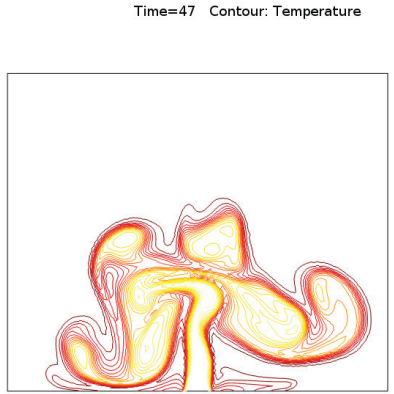

(d) $F r=2.5$
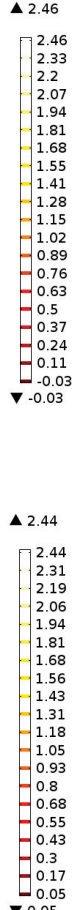

Fig. 8 Temperature field at time $\tau=47$ for plumes with $\operatorname{Re}=50, \operatorname{Pr}=7, \phi_{\text {in }}=2.5$ and (a) $\operatorname{Fr}=0.2$, (b) $F r=0.5$, (c) $F r=1$ and (d) $F r=2.5$

There is certainly scope for further computational investigations of plumes with buoyancy reversal due to nonlinear dependence of density on temperature. The range of Froude numbers should be increased, in particular to determine whether there is a different regime for $F r>2$. Higher Reynolds number should be investigated, both at transition to turbulence and in the fully turbulent case which is most relevant to environmental applications, with the aim of determining scalings of plume height against Reynolds number as found, for example, by Lin and Armfield [8]. Varying the Prandtl number may provide useful insight into our proposed explanation of the Froude number scaling of plume height. Different geometrical parameters of interest are, firstly, an axisymmetric source (although the flow would not be expected to remain axisymmetric); and secondly, a low-ceilinged domain so that the plume would reach the ceiling and start spreading below the ceiling while still positively buoyant, before mixing creates negative buoyancy so that the warm fluid then sinks to the floor.

\section{References}

1. Foster TD (1972) An analysis of the cabbeling instability in sea water. J Phys Oceanogr 2:294-301

2. Macqueen JF (1979) Turbulence and cooling water discharges from power stations. In: Harris CJ (ed) Mathematical Modelling of Turbulent Diffusion in the Environment. Academic Press, London, pp 379-437

3. Marmoush YR, Smith AA, Hamblin PF (1984) Pilot experiments on thermal bar in lock exchange flow. J Energy Eng - ASCE 110:215-227

4. Hoglund B, Spigarelli SA (1972) Studies of the sinking plume phenomenon. In: Proc 15th Conf Great Lakes Res. International Association of Great Lakes Research, Ann Arbor, pp 614-624 


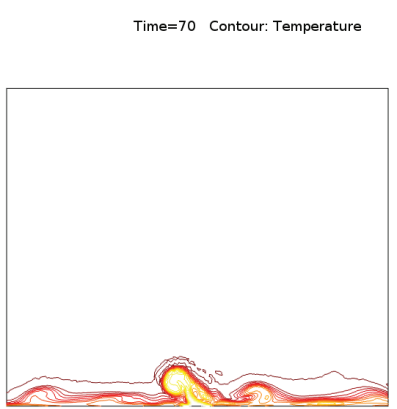

(a) $F r=0.2$

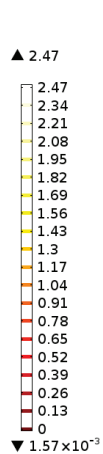

$\checkmark 1.57 \times 10^{-3}$

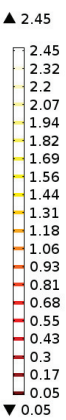

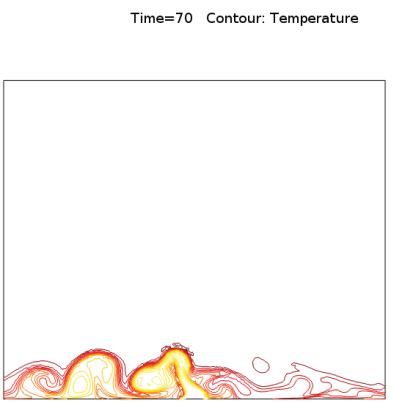

(b) $\mathrm{Fr}=0.5$
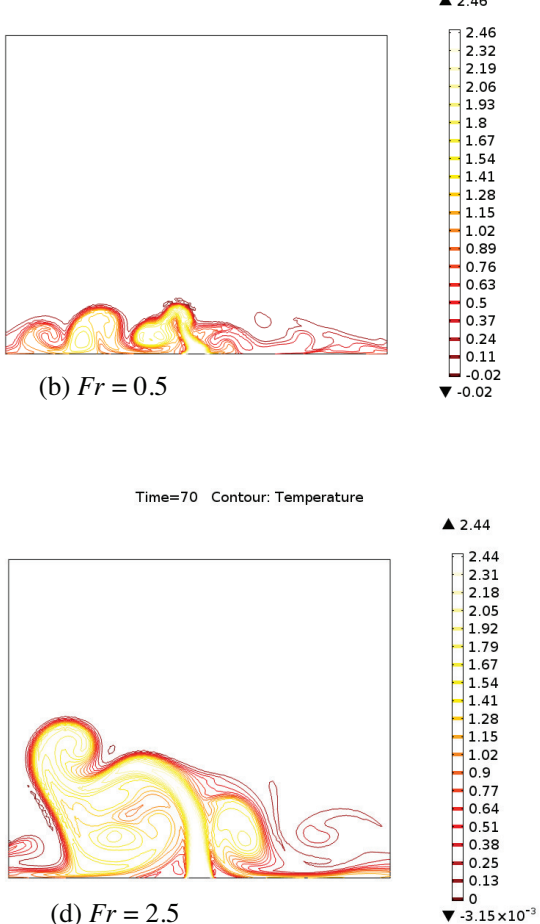

(d) $F r=2.5$

Fig. 9 Temperature field at time $\tau=70$ for plumes with $\operatorname{Re}=50, \operatorname{Pr}=7, \phi_{\text {in }}=2.5$ and (a) $F r=0.2$, (b) $F r=0.5$, (c) $F r=1$ and (d) $F r=2.5$

5. Bukreev VI, Gusev AV (2011) The effect of densification during mixing on the spreading of a vertical round jet. Dokl Earth Sci 439:1002-1005

6. Lin W, Armfield WS (2000) Direct simulation of weak axisymmetric fountains in a homogeneous fluid. J Fluid Mech 403:67-88

7. Lin W, Armfield WS (2000) Direct simulation of weak laminar plane fountains in a homogeneous fluid. Int J Heat Mass Tran 43:3013-3026

8. Lin W, Armfield WS (2003) The Reynolds and Prandtl number dependence of weak fountains. Comput Mech 31:379-389

9. Srinarayana N, McBain DG, Armfield WS, Lin W (2008) Height and stability of laminar plane fountains in a homogeneous fluid. Int J Heat Mass Tran 51:4717-4727

10. Srinarayana N, Armfield WS, Lin W (2013) Behaviour of laminar plane fountains with a parabolic inlet velocity profile in a homogeneous fluid. Int J Therm Sci 67:87-95

11. Williamson N, Srinarayana N, Armfield WS, McBain DG, Lin W (2008) Low-Reynolds-number fountain behaviour. J Fluid Mech 608:297-317

12. Srinarayana N, Williamson N, Armfield WS, Lin W (2010) Line fountain behaviour at low Reynolds number. Int J Heat Mass Tran 53:2065-2073

13. Burridge HC, Mistry A, Hunt GR (2015) The effect of source Reynolds number on the rise height of a fountain. Phys Fluids 27:047101

14. Turner JS (1966) Jets and plumes with negative or reversing buoyancy. J Fluid Mech 26:779-792

15. Caulfield C-CP, Woods AW (1995) Plumes with non-monotonic mixing behaviour. Geophys Astrophys Fluid Dyn 79:173-199

16. Morton BR, Taylor GI, Turner JS (1956) Turbulent gravitational convection from maintained and instantaneous sources. Proc R Soc Lond A 234:1-23

17. Kay A (2007) Warm discharges in cold fresh water: 1. Line plumes in a uniform ambient. J Fluid Mech 574:239-271

18. Moore DR, Weiss NO (1973) Nonlinear penetrative convection. J Fluid Mech 61:553-581 


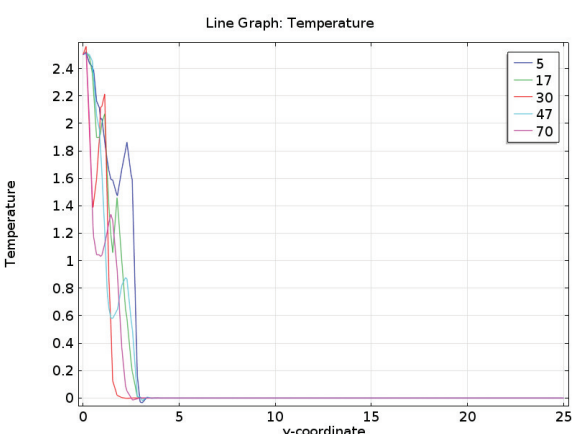

(a) $\mathrm{Fr}=0.2$

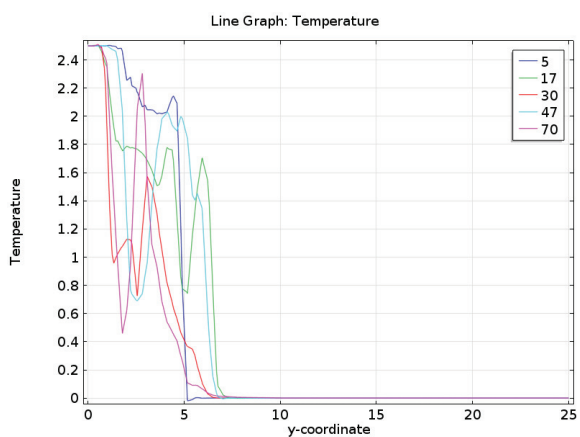

(c) $\mathrm{Fr}=1.0$

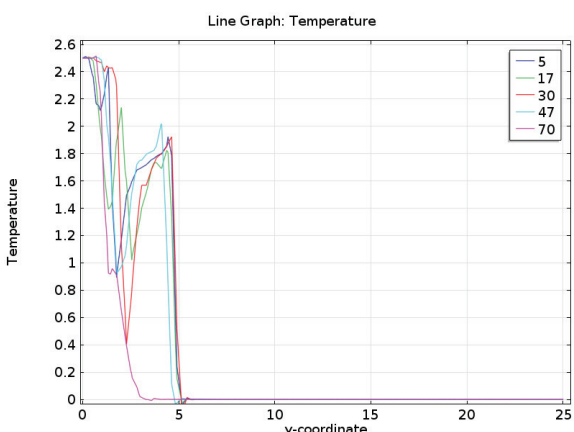

(b) $\mathrm{Fr}=0.5$

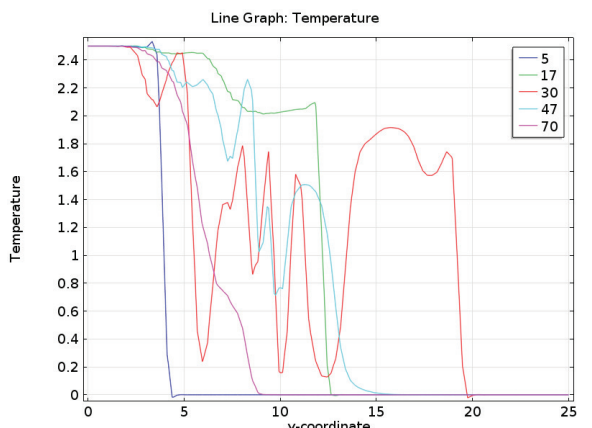

(d) $F r=2.5$

Fig. 10 Dimensionless centreline temperature distribution $\phi(0, y)$ at times $\tau=5,17,30,47,70$, for plumes with $R e=50, P r=7, \phi_{\text {in }}=2.5$ and (a) $F r=0.2$, (b) $F r=0.5$, (c) $F r=1$ and (d) $F r=2.5$

19. Oosthuizen PH, Paul JT (1996) A numerical study of the steady state freezing of water in an open rectangular cavity. Int J Numer Meth Heat Fluid Flow 6:3-16
20. COMSOL
Multiphysics
Cyclopedia:
The Finite
Element
Method.
https://www.comsol.com/multiphysics/finite-element-method . Accessed 28 April 2016

21. Vinoth BR, Panigrahi PK (2014) Characteristics of low Reynolds number non-Boussinesq fountains from non-circular sources. Phys Fluids 26:014106

22. Simpson JE (1982) Gravity currents in the laboratory, atmosphere, and ocean. Ann Rev Fluid Mech $14: 213-234$

23. Lin W, Armfield WS (2000) Very weak fountains in a homogeneous fluid. Numer Heat Transfer A 38:377-396 


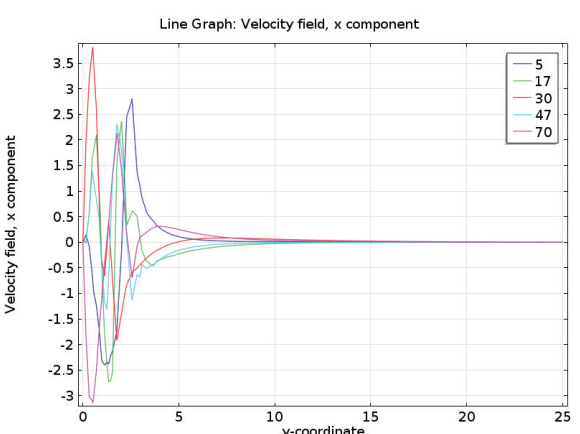

(a) $F r=0.2$

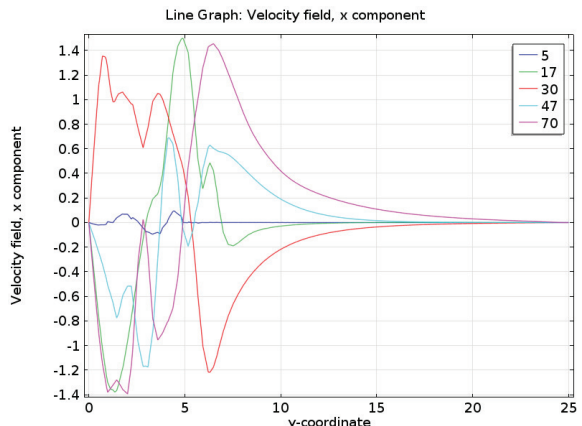

(c) $F r=1.0$

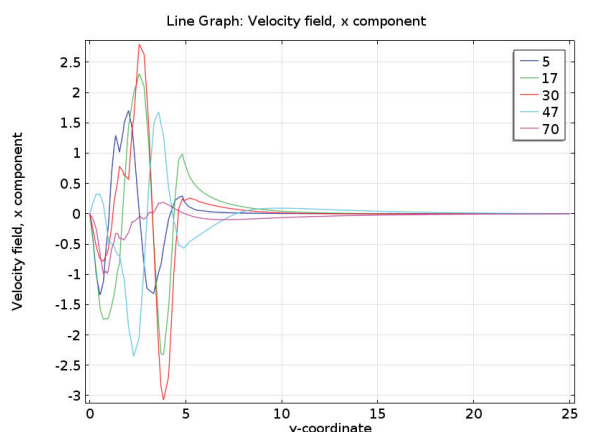

(b) $F r=0.5$

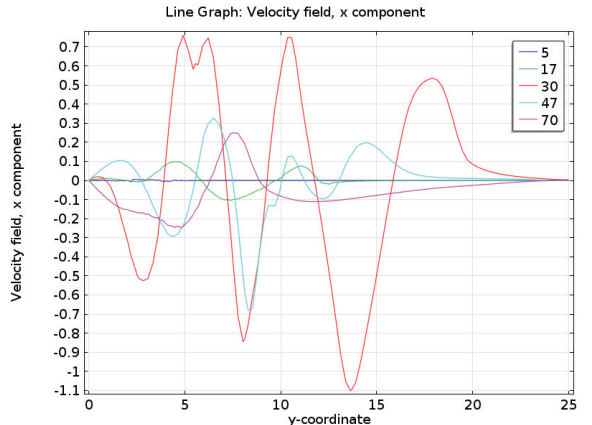

(d) $F r=2.5$

Fig. 11 Dimensionless centreline X-component velocity distribution $U(0, y)$ at times $\tau=5,17,30,47,70$, for plumes with $\operatorname{Re}=50, \operatorname{Pr}=7, \phi_{\text {in }}=2.5$ and (a) $F r=0.2$, (b) $F r=0.5$, (c) $F r=1$ and (d) $F r=2.5$ 


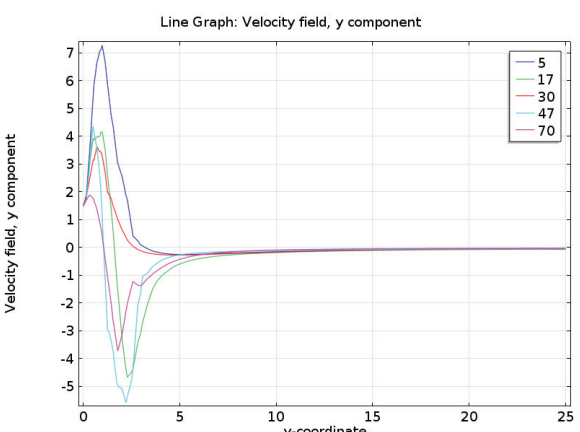

(a) $F r=0.2$

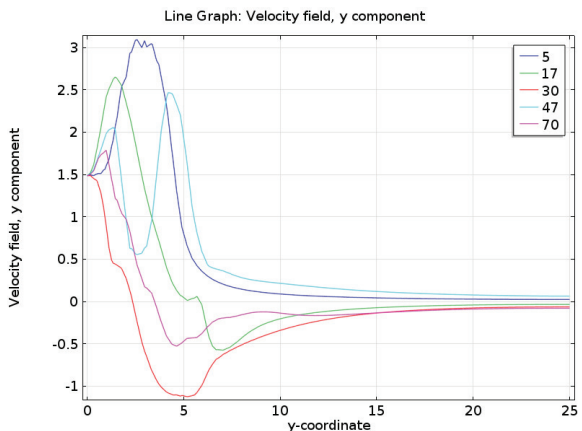

(c) $F r=1.0$

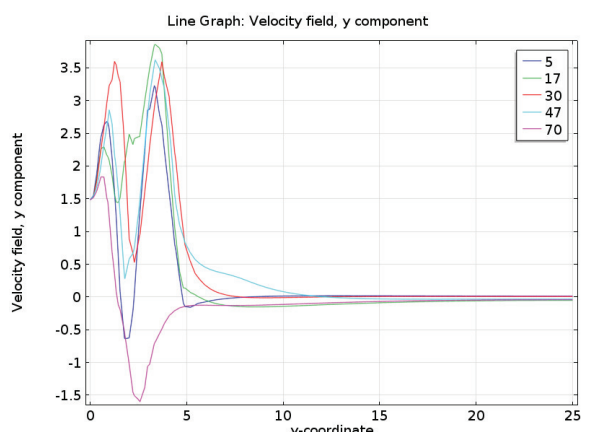

(b) $F r=0.5$

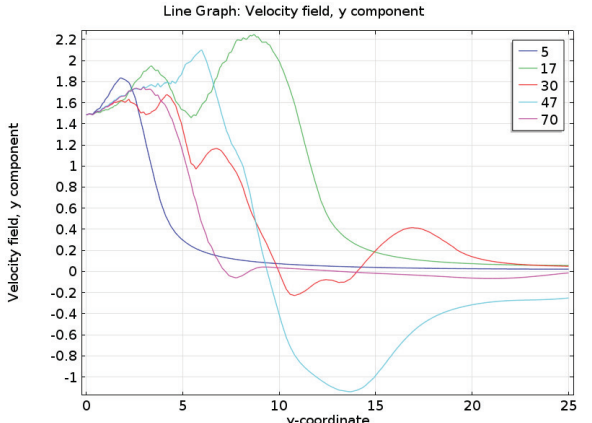

(d) $\mathrm{Fr}=2.5$

Fig. 12 Dimensionless centreline Y-component velocity distribution $V(0, y)$ at times $\tau=5,17,30,47,70$, for plumes with $R e=50, P r=7, \phi_{\text {in }}=2.5$ and (a) $F r=0.2$, (b) $F r=0.5$, (c) $F r=1$ and (d) $F r=2.5$

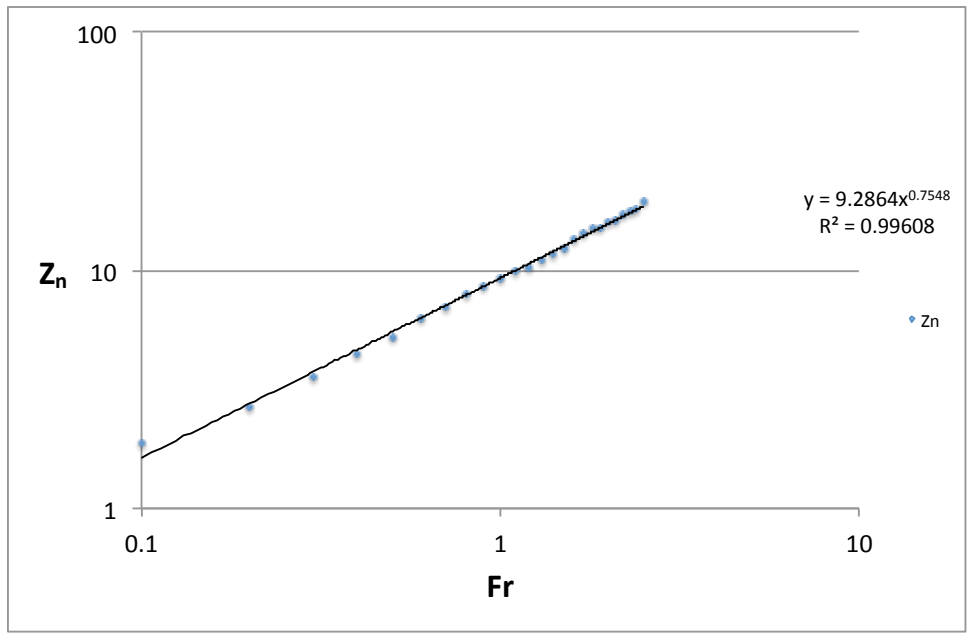

Fig. 13 Variation of maximum plume height with Froude number 


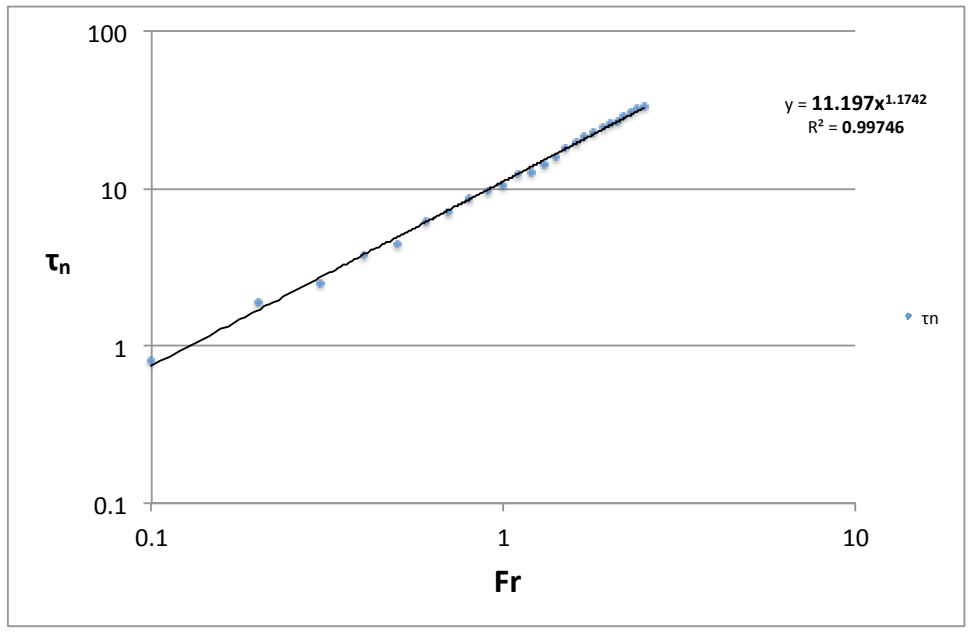

Fig. 14 Time taken for plume to reach maximum height, as a function of Froude number 